\title{
Nyquist-SEFDM: Pulse Shaped Multicarrier Communication with Sub-Carrier Spacing Below the Symbol Rate
}

\author{
(Invited Paper) \\ Tongyang $\mathrm{Xu}$ and Izzat Darwazeh \\ Department of Electronic and Electrical Engineering, University College London, London, UK \\ Email: t.xu@ee.ucl.ac.uk, i.darwazeh@ucl.ac.uk
}

\begin{abstract}
A new waveform design which simultaneously compresses bandwidth and suppresses out-of-band power leakage is studied in this work considering future 5th generation (5G) requirements. Thus, doubly created interference, coming from less than symbol rate packed sub-carriers and pulse shaping filters, is introduced. Therefore, this work, through using specially designed detectors, deals with the doubly created interference problem. It paves the way to non-orthogonal signal detection and non-orthogonal carrier aggregation (CA) system designs; both of importance to future wireless and wired communication systems.

Index Terms-Multicarrier communications, 5G, pulse shaping, spectral efficiency, OFDM, SEFDM, non-orthogonal.
\end{abstract}

\section{INTRODUCTION}

Many of today's communication systems utilize multicarrier transmission techniques as such techniques offer good spectrum utilization and are robust against degradation effects caused by multipath propagation. Most prominent of the multicarrier systems is the orthogonal frequency division multiplexing (OFDM) where the information signal is carried on parallel orthogonal sub-carriers with frequency separation equal to the symbol rate. OFDM is currently the system of choice for many wire and wireless transmission systems, most notably and currently as the downlink transmission system for the 4 th generation $(4 \mathrm{G})$ cellular system long term evolution (LTE) [1].

In future $5 \mathrm{G}$ networks, a thousand-fold wireless traffic increase is predicted from 2020, originating from over 50 billion connected devices [2]. Such massive device connection and communication will be associated with the continuously emerging internet of things (IoT). In order to support faster data speed and provide sufficient multiple access, new waveform designs are needed. The spectrum scarcity has led to the emergence of several spectrally efficient techniques [3][4], with efficiencies exceeding that of OFDM. Of these techniques, the non-orthogonal multicarrier system termed spectrally efficient frequency division multiplexing (SEFDM), initially proposed in 2003 [5], improves spectral efficiency by packing sub-carriers at frequency spacing below the orthogonality limit (i.e. below the symbol rate). Conceptually, this can be illustrated in Fig. 1 where the spectra of OFDM and
SEFDM are compared, clearly showing the bandwidth saving advantage of SEFDM relative to OFDM for the same number of sub-carriers and the same sub-carrier bandwidth or symbol rate. This is a result of sub-carriers overlapping in SEFDM at spacings below the symbol rate, effectively compressing subcarrier frequency spacing and leading to improved spectral efficiency, at the expense of self-created (yet deterministic) inter carrier interference (ICI) and increased complexity [6].

Recently, SEFDM has been experimentally implemented and validated in wireless and optical systems. In wireless systems, the SEFDM signal has been incorporated in a densely packed CA testbed [7] and a $3.75 \mathrm{Gbit} / \mathrm{s} 60 \mathrm{GHz}$ millimeter wave radio-over-fiber (RoF) testbed [8][9]. In optical systems, a $10 \mathrm{Gbit} / \mathrm{s}$ direct detection system [10] and a $24 \mathrm{Gbit} / \mathrm{s}$ coherent detection system [11] have been demonstrated. It was shown experimentally that not only better error performance is obtained when comparing SEFDM to OFDM systems with the same spectral efficiency but also higher data rates are achieved when considering the same occupied bandwidth for SEFDM and OFDM. A conceptually similar technique, termed faster than Nyquist (FTN) [4] has been extensively studied. The capacity merits of this technique have been verified through information theoretic studies in [12] and more recently for SEFDM in [13]. For such non-orthogonal multicarrier systems, the capacity benefits and spectral efficiency, and wireless channel tolerant nature is what is making them attractive for use in future communication systems such as 5G [14][15][16][17].

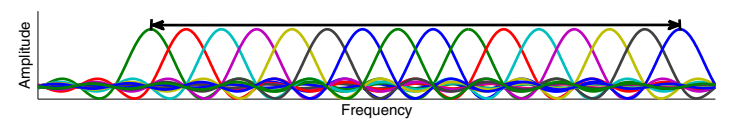

(a) OFDM Spectrum.

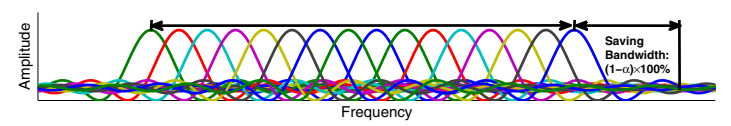

(b) SEFDM Spectrum with bandwidth compression factor $\alpha=0.8$.

Figure 1. Spectra of 16 overlapped sub-carriers for OFDM and SEFDM.

As mentioned in [7], integrating more signal bands in 
a given bandwidth provides an efficient way to tackle the shortage of radio spectrum. However, the use of rectangular pulses in typical SEFDM systems results in high out-of-band power leakage and consequently in interference to adjacent frequency bands. Thus, a frequency gap has to be used as a protection gap which reduces spectral efficiency. Recently, a spectrally efficient concept, employing an root raised cosine (RRC) pulse shaping filter for each sub-carrier, demonstrated significant reduction of out-of-band power leakage, when applied to the multicarrier system termed generalized frequency division multiplexing (GFDM) [18]. However, because of the RRC filter shaping effects, ICI is introduced. Work in [18] has verified that with the assistance of a successive interference cancellation scheme, GFDM can achieve the same BER performance as that of the rectangular pulse shaped OFDM. It should be noted that contrary to SEFDM, the subcarrier spacing of the GFDM system is still equal to the symbol rate. Since the out-of-band power is significantly suppressed in GFDM [15], it would be spectrally advantageous to reduce the sub-carrier spacing below the symbol rate and reduce the outof-band power at the same time. Therefore, the motivation for this design study of Nyquist-SEFDM is to create a spectrally efficient system with minimum out-of-band power.

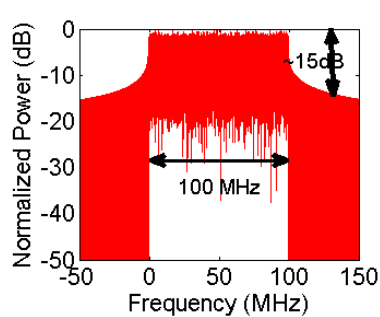

(a) Rectangular shaped OFDM.

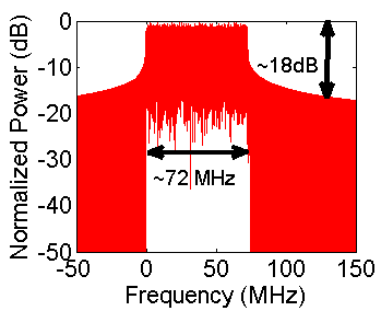

(c) Rectangular shaped SEFDM.

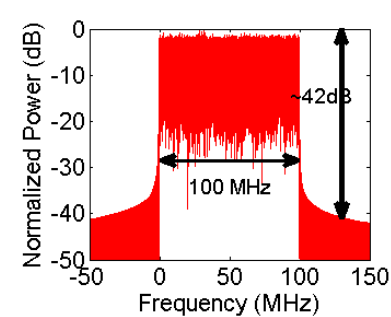

(b) RRC shaped OFDM.

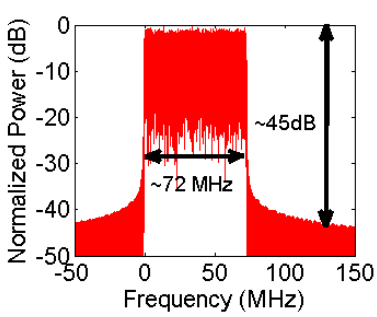

(d) RRC shaped SEFDM.
Figure 2. Bandwidth saving and out-of-band power reduction comparisons for various signals. Using the same number of sub-carriers, the bandwidth is compressed by $28 \%$ in SEFDM systems, the roll-off factor of the RRC filter is $\beta=0.5$ and the filter is truncated to six symbols.

The idea of the Nyquist-SEFDM is illustrated in Fig. 2 where spectra of different systems are illustrated for the purpose of comparison. Fig. 2(a) is a typical OFDM spectrum with rectangular pulses, carrying 100 Msymbols/s, over 100 $\mathrm{MHz}$ bandwidth. The out-of-band power is approximately 15 $\mathrm{dB}$ below the spectral peak. Using RRC filtering, in Fig. 2(b), such out-of-band power leakage is reduced by a further $27 \mathrm{~dB}$. Finally, for the same data rate, a rectangular pulse shaped SEFDM is shown in Fig. 2(c) where the bandwidth is compressed to $72 \mathrm{MHz}$ while the out-of-band power is reduced to $-18 \mathrm{~dB}$. This indicates that SEFDM is better than OFDM in both bandwidth saving and the out-of-band power suppression. Furthermore, the Nyquist-SEFDM spectrum is shown in Fig. 2(d) where the out-of-band power leakage is around $-45 \mathrm{~dB}$ (i.e. the lowest one) over a $72 \mathrm{MHz}$ compressed bandwidth.

\section{PRINCIPLE OF NYQUIST-SEFDM}

\section{A. SEFDM System Model}

In this work, the discrete sampled version of the SEFDM signal obtained from [19] is studied and shown in (1)

$$
X[k]=\frac{1}{\sqrt{Q}} \sum_{n=0}^{N-1} s_{n} \exp \left(\frac{j 2 \pi n k \alpha}{Q}\right)
$$

where $X[k]$ is the $k^{t h}$ time sample with $k=[0,1, \ldots, Q-1]$, $N$ is the number of sub-carriers, $Q=\rho N$ and $\rho \geq 1$ is the oversampling factor, $\frac{1}{\sqrt{Q}}$ is a normalization scaling factor and $s_{n}$ is a QAM symbol of duration $T$, modulated on the $n^{\text {th }}$ sub-carrier. $\alpha$ is bandwidth compression factor defined as $\alpha=\Delta f T$, where $\Delta f$ denotes the sub-carrier spacing. It is evident that (1) cannot be straightforwardly implemented using an IFFT due to the introduction of $\alpha$. Work in [19] detailed and compared methods that effectively employ different IFFT architectures for SEFDM signal generation.

\section{B. Transmitter of Nyquist-SEFDM}

A block diagram of the Nyquist-SEFDM transmitter is depicted in Fig. 3. Two scenarios are studied in this work. In scenarios A (uncoded scheme), the binary bit stream is directly mapped into QAM symbols. In scenarios B (coded scheme) [3], the binary bit stream is firstly convolutionally encoded in the encoder and then permuted through a random interleaver $\Pi$. The detailed information of the encoder and the random interleaver is given in [3]. After that, complex symbols are obtained in the QAM mapper. Further processing is the same for both scenarios. After serial to parallel conversion, one data stream $S$ is divided into $N$ parallel branches; the $n^{\text {th }}$ branch is denoted as $S_{n}$. In one branch, the complex data stream $S_{n}=\left[s_{n, 0}, s_{n, 1}, \ldots\right]$ is up sampled by a factor of $Q$ leading to a new complex data vector $W_{n}=\left[w_{n, 0}, w_{n, 1}, \ldots\right]$ which is defined as:

$$
W_{n}[k]= \begin{cases}S_{n}\left[\frac{k}{Q}\right] & k \bmod Q=0 \\ 0 & \text { otherwise }\end{cases}
$$

Thereafter, $W_{n}$ is convolved with $g(m)$, which is the impulse response of each of the RRC filters, truncated to $L$ symbols where each one contains $Q$ samples. Therefore. The order of each filter is $U=Q \times L$ indicating $m=[0,1, \ldots, U-1]$ and the output of each is $Y_{n}$, expressed as

$$
Y_{n}=W_{n} * g(m)
$$

where $*$ denotes convolution. After filtering, the pulse shaped data is up converted to sub-carrier frequency $f_{n}$.

Unlike rectangular pulse shaped systems, clearly the samples of one symbol no longer have equal values in the RRC 


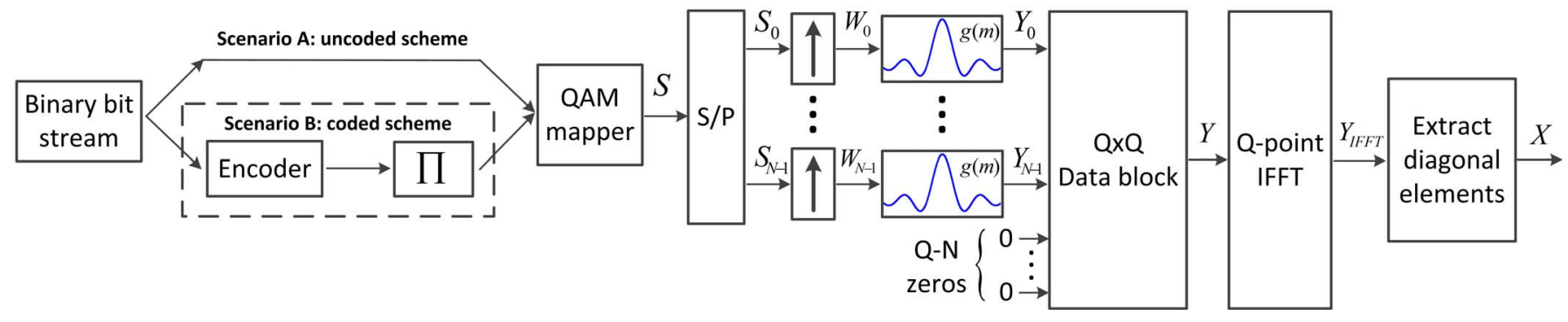

Figure 3. Block diagram of the Nyquist-SEFDM transmitter.

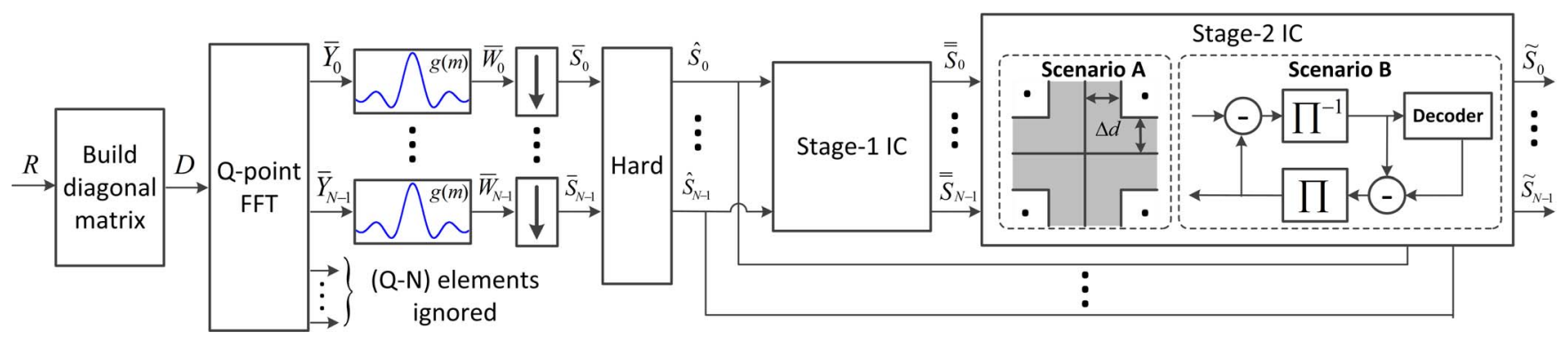

Figure 4. Block diagram of the Nyquist-SEFDM receiver.

filtered systems. Therefore, in Fig. 3, a $Q \times Q$ data block is introduced to buffer $Q$ samples from each branch every $T$ period. Assuming the data stream after the pulse shaping operation on the $n^{t h}$ branch is $Y_{n}=\left[y_{n, 0}, y_{n, 1}, \ldots\right]$, the $Q \times Q$ data matrix is defined as

$$
\mathbf{Y}=\left[\begin{array}{cccc}
y_{0,0} & y_{0,1} & \cdots & y_{0, Q-1} \\
y_{1,0} & y_{1,1} & \cdots & y_{1, Q-1} \\
\vdots & \vdots & \vdots & \vdots \\
y_{N-1,0} & y_{N-1,1} & \cdots & y_{N-1, Q-1} \\
0 & 0 & \cdots & 0 \\
\vdots & \vdots & \vdots & \vdots \\
0 & 0 & \cdots & 0
\end{array}\right]
$$

where $y_{n, k}$ indicates the $k^{t h}$ sample on the $n^{\text {th }}$ sub-carrier frequency (branch). One row, $\left[y_{n, 0}, y_{n, 1}, \ldots, y_{n, Q-1}\right]$ contains time samples that will modulate one specific sub-carrier of frequency $f_{n}$ and is defined as one sample vector. One column, the frequency vector $\left[y_{0, k}, y_{1, k}, \ldots, y_{N-1, k}, \ldots, 0\right]^{t r}$ of the data matrix contains time samples to be modulated at all carrier frequencies. The notation $t r$ indicates the transpose operation. It should be noted that $Q-N$ additional branches are introduced via padding $Q-N$ zeros at the end of each frequency vector.

The up conversion of each branch is done by

$$
X[k]=\sum_{n=0}^{Q-1} y_{n, k} \cdot \exp \left(\frac{j 2 \pi n k \alpha}{Q}\right)
$$

where $n, k=[0,1, \ldots, Q-1]$. The operation in (5) can be efficiently realized by IFFT either for OFDM $(\alpha=1)$ or SEFDM $(\alpha<1)$ [19]. In a rectangular pulse shaped system, the size of the data block is $Q \times 1$, since the elements of the sample vector $\left[y_{n, 0}, \ldots, y_{n, Q-1}\right]$ have constant amplitude. Therefore, a single IFFT is operated every $T$ time period. However, for the $Q \times Q$ data matrix, a single IFFT has to be activated every $T / Q$ time period, requiring a higher processing speed IFFT. Alternatively, a single higher speed IFFT can be replaced by $Q$ parallel lower speed IFFTs, which relaxes the requirement of processing speed at the expense of increased resource consumption.

It should be noted that the IFFT returns results for each column of the data block matrix. Therefore, a $Q \times Q$ matrix $Y_{I F F T}$ is obtained after the IFFT processing. However, the useful data is only the diagonal elements of the matrix. Thus, extracting the diagonal elements from $Y_{I F F T}$ is required to give the transmitted signal $X$ as

$$
X=\operatorname{diagonal}\{\operatorname{IFFT}(\mathbf{Y})\}=\operatorname{diagonal}\{\mathbf{F Y}\}
$$

where the right hand side of (6) simply means that the process is equivalent to extracting the diagonal elements from sampled sub-carriers matrix $\mathbf{F}$ modulated by $\mathbf{Y}$.

Alternative and faster techniques for generating the same signals are given in [19] and utlize a pruned IFFT algorithm that can remove the redundant operations leading to reduced complexity.

\section{Receiver of Nyquist-SEFDM}

To illustrate the concept, a simple AWGN channel is used in this work. A block diagram of the Nyquist-SEFDM receiver 
is shown in Fig. 4. After propagating in an AWGN channel, the received signal is expressed as

$$
R=X+Z
$$

where $R=\left[r_{0}, r_{1}, \ldots r_{Q-1}\right]$ is the received signal and $Z$ denotes AWGN.

As shown at the transmitter side, the transmitted symbols $X$ are the diagonal elements extracted from the $Q \times Q$ IFFT operated matrix $Y_{I F F T}$. Therefore, before the FFT operation at the receiver side, a diagonal matrix such as (8) is required to be built for every $Q$ samples.

$$
\mathbf{D}=\left[\begin{array}{cccc}
r_{0} & 0 & \cdots & 0 \\
\vdots & r_{1} & \cdots & 0 \\
\vdots & \vdots & \ddots & \vdots \\
0 & 0 & \cdots & r_{Q-1}
\end{array}\right]
$$

Then, the demodulation can be expressed as:

$$
\bar{y}_{n, k}=r_{k} \cdot \exp \left(\frac{-j 2 \pi n k \alpha}{Q}\right)
$$

where $\bar{y}_{n, k}$ is an element of matrix $\overline{\mathbf{Y}}$ and $n, k=[0,1, \ldots, Q-$ 1]. It should be noted that a Q-point FFT can be used in (9) either for OFDM $(\alpha=1)$ or SEFDM $(\alpha<1)$ [19]. Furthermore, (9) can be rearranged in a matrix form as:

$$
\overline{\mathbf{Y}}=F F T(\mathbf{D})=\mathbf{F}^{*} \mathbf{D}
$$

where $\mathbf{F}^{*}$ is the conjugate of the sub-carrier matrix $\mathbf{F}$. It should be noted that the computational complexity can be reduced by skipping zero elements within the matrix $\mathbf{D}$ using the pruned FFT [19]. After the FFT, the last $Q-N$ elements of each output vector are ignored. Then, RRC filters identical to those used at the transmitter with $g(m)$, operate on the signals of each branch as in (11). This is followed by the down sampling operation as shown in (12).

$$
\begin{gathered}
\bar{W}_{n}=\bar{Y}_{n} * g(m) \\
\bar{S}_{n}[k]=\bar{W}_{n}[Q \times k]
\end{gathered}
$$

Utilizing a hard decision module, initial estimates $\hat{S}$ are obtained.

ISI and ICI exist in Nyquist-SEFDM due to the combine use of the RRC pulse shaping filter and the less than symbol rate packed sub-carriers. In order to obtain better BER performance, interference cancellation is required to remove the ICI effect. In a rectangular pulse shaped SEFDM system, interference to one sub-carrier comes from all other subcarriers. However, for an RRC pulse shaped SEFDM system, due to the reduced out-of-band power, interference is mostly limited to neighbouring two sub-carriers resulting in relatively simplified interference cancellation.

The interference cancellation (IC) includes two stages. The first stage is a general IC module (stage-1 IC) and the second stage is a specially designed module (stage-2 IC) for NyquistSEFDM. Interference imposed on the $n^{\text {th }}$ branch comes from the $(n-1)^{t h}$ and the $(n+1)^{t h}$ branches. Data streams $\hat{S}_{n-1}$ and $\hat{S}_{n+1}$ are extracted after the hard decision. After the up sampling and pulse shaping operations, two sequences are up converted to frequencies $f_{n-1}$ and $f_{n+1}$ multiplying with $e^{\frac{j 2 \pi(n-1) k \alpha}{Q}}$ and $e^{\frac{j 2 \pi(n+1) k \alpha}{Q}}$, respectively. This process is to model the interference superimposed on the targeted signal on the $n^{t h}$ branch. Therefore, before the demodulation of the received signal, $R$, interference is subtracted as

$$
R_{n}=R-I_{n-1}-I_{n+1}
$$

where $R_{n}$ is the interference cancelled signal on the $n^{\text {th }}$ branch, $I_{n-1}$ is the modulated signal from the $(n-1)^{t h}$ branch and $I_{n+1}$ is the $(n+1)^{t h}$ branch. After that, the interference cancelled data stream is down converted to baseband by multiplying $R_{n}$ with $e^{\frac{-j 2 \pi n k \alpha}{Q}}$. After the subsequent pulse shaping and down sampling operations, the recovered data stream $\overline{\bar{S}}_{n}$ is obtained. The same interference cancellation operation inside the stage-1 IC is repeated for the rest of $N-1$ branches. The stage- 1 IC is very similar to the two-sided successive interference cancellation (SIC) proposed for GFDM [18]. However, unlike GFDM, the additional interference in SEFDM has to be removed using a specially designed SEFDM detector termed stage-2 IC.

Two scenarios are demonstrated in Fig. 4. The iterative soft demapping scheme termed iterative detection (ID) in [20] is evaluated as the scenario A, with uncoded data. An adaptive decision threshold $\Delta d$ is preferred. The principle of the ID scheme is firstly to decide less distorted symbols and then recover highly distorted symbols on the basis of the decided ones. It is concluded from [20] that the decision of one symbol is dependent on the recovered symbols from previous iterations. In order to improve the reliability of a decision after each iteration, coded data is used with Turbo equalizer [21][22] in scenario B, to improve the reliability of symbol decisions iteratively. Thus, BER performance is improved.

\section{RESULTS}

This work aims to demonstrate the concept of the NyquistSEFDM. Therefore, a Nyquist-SEFDM system with 64 data sub-carriers and 4QAM modulation scheme is investigated in an AWGN channel. Two specific bandwidth compression factors are selected according to the work [7]. For a more realistic wireless environment like multipath fading, an SEFDM experimental testbed has been recently reported in [7].

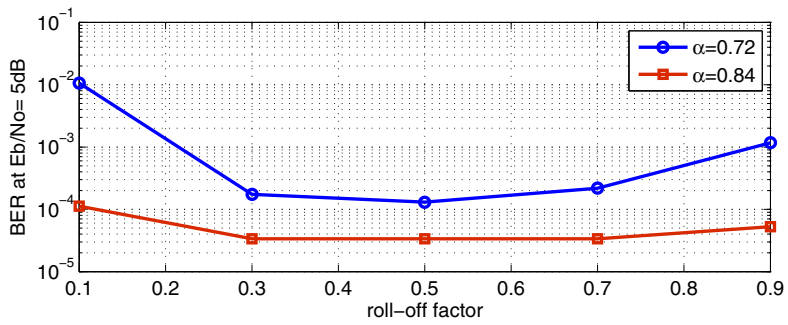

Figure 5. BER versus roll-off factors $\beta$. 
The RRC filter design is beyond the scope of this work. But it is necessary to evaluate the effect of different roll-off factors. The filter in this work is truncated to six symbols. In Fig. 5 , the RRC pulse with $\beta=0.5$ has the best performance. The performance becomes worse at both high and low $\beta$. At high $\beta$, more excess bandwidth is introduced leading to increased ICI. At low $\beta$, more ISI is introduced due to the filter truncation. Therefore, in the following simulations, the roll-off factor $\beta$ is set to be 0.5 .

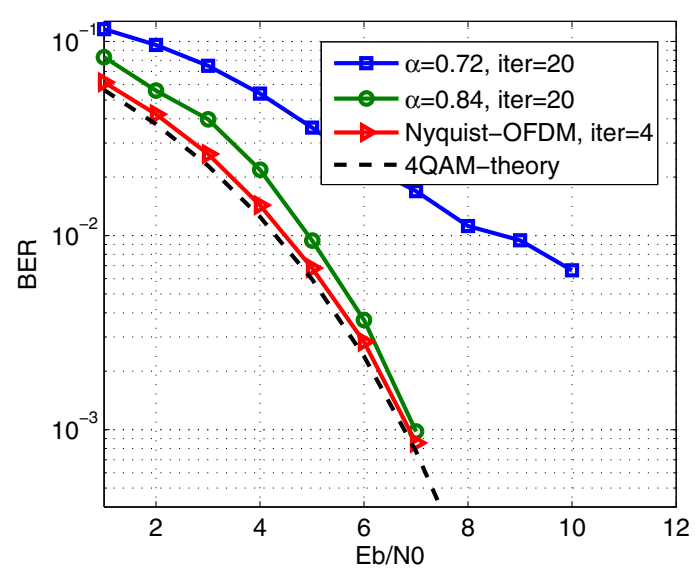

(a) BER performance.

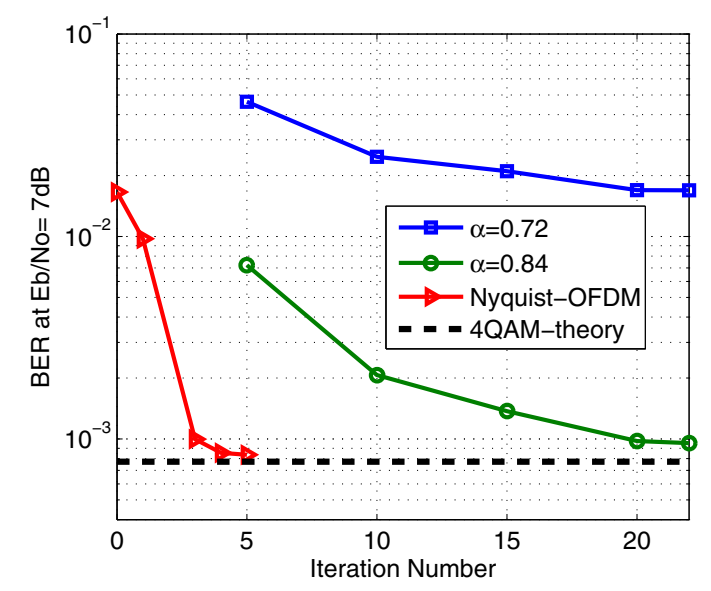

(b) Iteration performance.

Figure 6. Performance (scenario A) for different pulse shaped systems.

Due to the use of RRC filter, interference is introduced in both Nyquist-OFDM and Nyquist-SEFDM systems. It is shown in Fig. 6(a) that four iterations are needed for the uncoded Nyquist-OFDM system to get converged performance. For Nyquist-SEFDM, more iterations are required to cope with the additional interference resulting from extra interference introduced by the closer packing of sub-carriers. The iteration numbers for different Nyquist-SEFDM systems are investigated in Fig. 6(b) where with 20 iterations and $\alpha=0.84$, the performance approaches the theoretical one at around $E_{b} / N_{o}=7 \mathrm{~dB}$. However, for $\alpha=0.72$, even with 20 iterations, the performance is far away from the theoretical 4QAM one. This

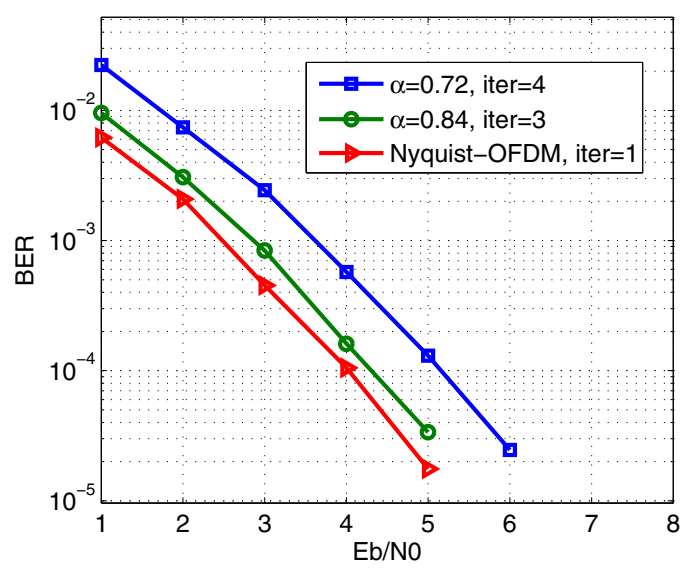

(a) BER performance.

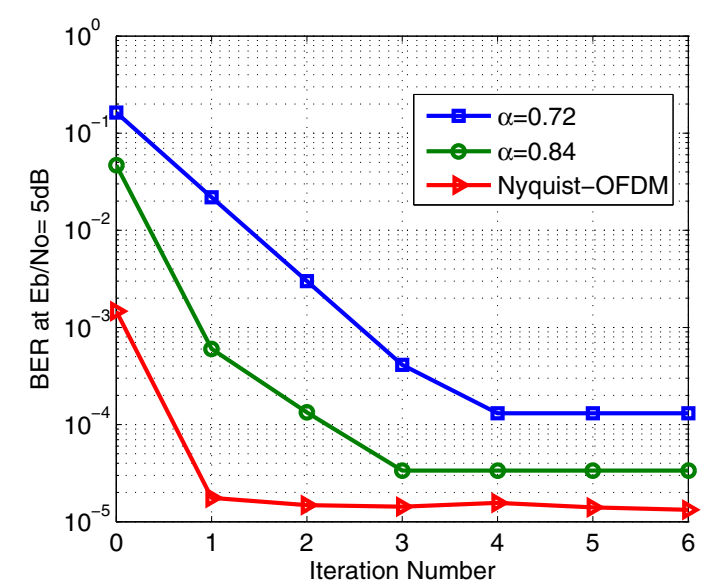

(b) Iteration performance.

Figure 7. Performance (scenario B) for different pulse shaped systems.

is due to the fact that with smaller $\alpha$ (i.e. higher bandwidth compression), more ICI is introduced in the system. This may be taken as a limitation of uncoded Nyquist-SEFDM when lower values of $\alpha$ are considered.

The coded technique is studied and results are evaluated in Fig. 7. It is apparent that for both Nyquist-OFDM and NyquistSEFDM systems, the required iteration numbers are reduced significantly. This is due to the fact that more reliable estimates are obtained after each iteration leading to an improved convergence speed. It is apparent that at $\mathrm{BER}=3 \times 10^{-5}$ the performance gap between the $\alpha=0.84$ and the Nyquist-OFDM one is approximately $0.3 \mathrm{~dB}$. For $\alpha=0.72$, the gap is $1.2 \mathrm{~dB}$.

\section{CONCLUSIONS}

The new waveform termed Nyquist-SEFDM, reported in this work, can simultaneously save bandwidth and reduce out-of-band power leakage. Bandwidth saving is achieved by packing sub-carriers at frequencies below the symbol rate. Spectral efficiency is improved at the cost of self-created ICI. The out-of-band power suppression is achieved by shaping each sub-carrier with an RRC filter, which introduces new 
interference. The doubly created interference and its effect are studied under two scenarios. One uses uncoded data and the other applies coding and Turbo equalization. Results indicate that the uncoded iterative detection can effectively remove interference at the expense of high iteration numbers. With the coded Turbo equalization scheme, signal detection is simplified with fewer iterations. Altogether, the signal studies show suitability for future communication systems where spectral efficiency and carrier aggregation are required to allow high data rate communication in an increasingly congested spectrum.

\section{REFERENCES}

[1] 3GPP TS 36.300 version 8.12.0 Release 8, "Evolved universal terrestrial radio access (E-UTRA) and evolved universal terrestrial radio access network (E-UTRAN); overall description; stage 2 (release 8)," April 2010.

[2] H. A. U. Mustafa, M. A. Imran, M. Z. Shakir, A. Imran, and R. Tafazolli, "Separation framework: An enabler for cooperative and D2D communication for future 5G networks," IEEE Communications Surveys Tutorials, vol. 18, no. 1, pp. 419-445, Firstquarter 2016.

[3] T. Xu and I. Darwazeh, "Spectrally efficient FDM: Spectrum saving technique for 5G?" in $5 G$ for Ubiquitous Connectivity (5GU), 2014 1st International Conference on, Nov 2014, pp. 273-278.

[4] J. Anderson, F. Rusek, and V. Öwall, "Faster-than-Nyquist signaling," Proceedings of the IEEE, vol. 101, no. 8, pp. 1817-1830, 2013.

[5] M. Rodrigues and I. Darwazeh, "A spectrally efficient frequency division multiplexing based communications system," in Proc. 8th Int. OFDM Workshop, Hamburg, 2003, pp. 48-49.

[6] I. Kanaras, A. Chorti, M. Rodrigues, and I. Darwazeh, "Spectrally efficient FDM signals: Bandwidth gain at the expense of receiver complexity," in Communications, 2009. ICC '09. IEEE International Conference on, June 2009, pp. 1-6.

[7] T. Xu and I. Darwazeh, "Bandwidth compressed carrier aggregation," in IEEE ICC 2015 - Workshop on $5 G$ \& Beyond - Enabling Technologies and Applications (ICC'15 - Workshops 23), London, United Kingdom, Jun. 2015, pp. 1107-1112.

[8] S. Mikroulis, T. Xu, and I. Darwazeh, "Practical demonstration of spectrally efficient FDM millimeter-wave radio over fiber systems for 5G cellular networking," in Proc. SPIE, vol. 9772, 2016, pp. 97720I1-97 720I-8.

[9] T. Xu, S. Mikroulis, J. E. Mitchell, and I. Darwazeh, "Bandwidth compressed waveform for $60 \mathrm{GHz}$ millimeter-wave radio over fiber experiment," Journal of Lightwave Technology, 2016.

[10] I. Darwazeh, T. Xu, T. Gui, Y. Bao, and Z. Li, "Optical SEFDM system; bandwidth saving using non-orthogonal sub-carriers," Photonics Technology Letters, IEEE, vol. 26, no. 4, pp. 352-355, Feb 2014.

[11] D. Nopchinda, T. Xu, R. Maher, B. Thomsen, and I. Darwazeh, "Dual polarization coherent optical spectrally efficient frequency division multiplexing," Photonics Technology Letters, IEEE, vol. 28, no. 1, pp. 83-86, Jan 2016.

[12] F. Rusek and J. B. Anderson, "Constrained capacities for faster-thanNyquist signaling," IEEE Transactions on Information Theory, vol. 55, no. 2, pp. 764-775, Feb 2009.

[13] D. Rainnie, Y. Feng, and J. Bajcsy, "On capacity merits of spectrally efficient FDM," in Military Communications Conference, MILCOM 2015 - 2015 IEEE, Oct 2015, pp. 581-586.

[14] J. Andrews, S. Buzzi, W. Choi, S. Hanly, A. Lozano, A. Soong, and J. Zhang, "What will 5G be?" Selected Areas in Communications, IEEE Journal on, vol. 32, no. 6, pp. 1065-1082, June 2014.

[15] M. Danneberg, R. Datta, and G. Fettweis, "Experimental testbed for dynamic spectrum access and sensing of 5G GFDM waveforms," in Vehicular Technology Conference (VTC Fall), 2014 IEEE 80th, Sept 2014, pp. 1-5.

[16] I. Darwazeh, T. Xu, and R. C. Grammenos, Signal Processing for 5G: Algorithms and Implementations. Wiley, 2016, ch. Bandwidth Compressed Multicarrier Communication:SEFDM, p. to appear.

[17] I. Darwazeh, R. C. Grammenos, and T. Xu, Key Enabling Technologies for $5 G$ Mobile Communications. Springer, 2016, ch. Spectrally Efficient Frequency Division Multiplexing for 5G, p. to appear.
[18] R. Datta, N. Michailow, M. Lentmaier, and G. Fettweis, "GFDM interference cancellation for flexible cognitive radio PHY design," in Vehicular Technology Conference (VTC Fall), 2012 IEEE, Sept 2012, pp. 1-5.

[19] P. Whatmough, M. Perrett, S. Isam, and I. Darwazeh, "VLSI architecture for a reconfigurable spectrally efficient FDM baseband transmitter," Circuits and Systems I: Regular Papers, IEEE Transactions on, vol. 59, no. 5, pp. 1107-1118, May 2012.

[20] T. Xu, R. C. Grammenos, F. Marvasti, and I. Darwazeh, "An improved fixed sphere decoder employing soft decision for the detection of nonorthogonal signals," Communications Letters, IEEE, vol. 17, no. 10, pp. 1964-1967, October 2013.

[21] J. Hagenauer, "The turbo principle: tutorial introduction and state of the art," in Proc. Int. Symp. Turbo Codes, Sept 1997, pp. 1-11.

[22] T. Xu and I. Darwazeh, "A soft detector for spectrally efficient systems with non-orthogonal overlapped sub-carriers," Communications Letters, IEEE, vol. 18, no. 10, pp. 1847-1850, Oct 2014. 